\title{
Dynamo action in flows with cat's eyes
}

\author{
ALICE COURVOISIER †, ANDREW D. GILBERT*† \\ and YANNICK PONTY§ \\ $\dagger$ Department of Applied Mathematics, University of Leeds, UK \\ $\$$ Department of Mathematical Sciences, University of Exeter, UK \\ §Observatoire de la Côte d'Azur, Nice, France
}

(Received 18 April 2005; in final form 19 August 2005)

\begin{abstract}
This paper considers dynamo action in steady fluid flows having a cat's eye stream line topology. The flows considered are two-dimensional, depending on $x$ and $y$ only, but have all three components of velocity and hence can act as kinematic dynamos. The limit of large magnetic Reynolds number $(R)$ is considered and the numerical results are presented for the different types of magnetic mode that can be amplified. In particular, a new class of modes, which are termed cat's eye modes, is identified. These modes consist of sheets of field that lie along the separatrices and hyperbolic points that define a single row of as cat's eyes. They have growth rates that fall off very slowly with a large $R$, and their wavenumber $k$ in the $z$ direction scales approximately as $\sqrt{R}$. The fluid flow amplifies these magnetic fields by a variant of the stretch-fold-shear mechanism, acting in a steady flow.
\end{abstract}

Keywords: Dynamo; Cat's eye flow; Induction equation; Magnetic field; Alpha effect

\section{Introduction}

The aim of this article is to study dynamo action in steady two-dimensional flows $\mathbf{u}(x, y)$ for which the stream lines possess a cat's eye topology. When the flow is projected onto the $(x, y)$-plane, as shown in figure 1, some stream lines form closed eddies, the eyes of the cat, while others are open, forming channels; in between is a network of separatrices and hyperbolic stagnation points. Such flows are commonly generated by hydrodynamic instabilities, an example being the Ekman instability. In a rotating fluid system, an Ekman layer can become hydrodynamically unstable at a sufficiently high Reynolds number based on the Ekman layer thickness. Rolls appear within the fluid flow, which takes a two-dimensional form and is steady in a suitably translating frame.

These steady two-dimensional flows, which possess all three components of velocity, $\mathbf{u}=\left(u_{1}, u_{2}, u_{3}\right)(x, y)$, are sufficiently complicated to function as kinematic dynamos.

*Corresponding author. Email: A.D.Gilbert@exeter.ac.uk 


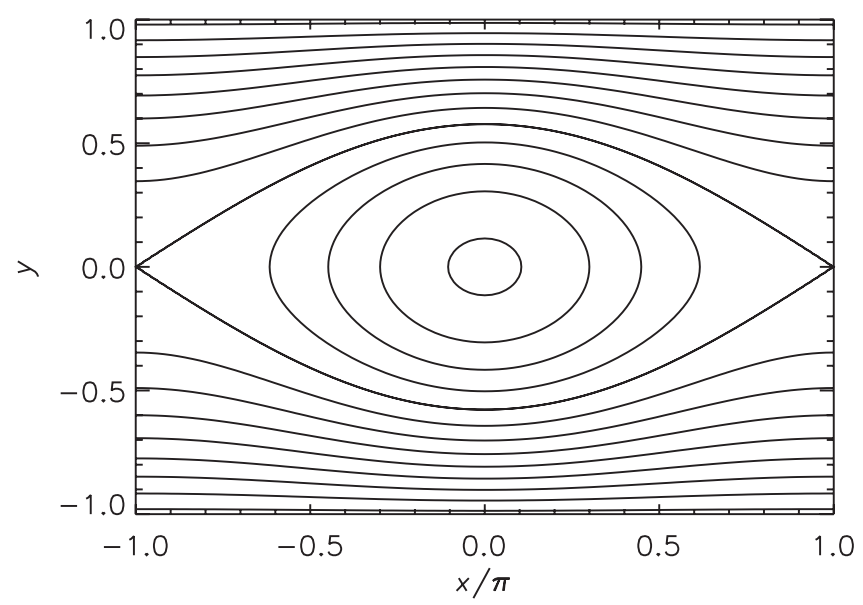

Figure 1. Stream lines of constant $\psi(x, y)$ are shown for the flow (2.4). The flow is periodic in the $x$-direction.

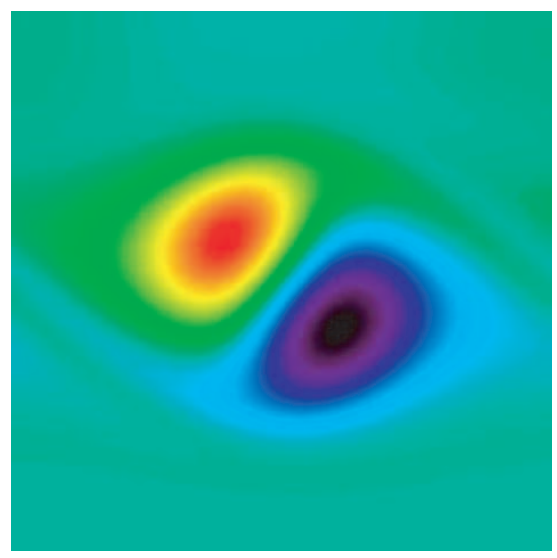

(a)

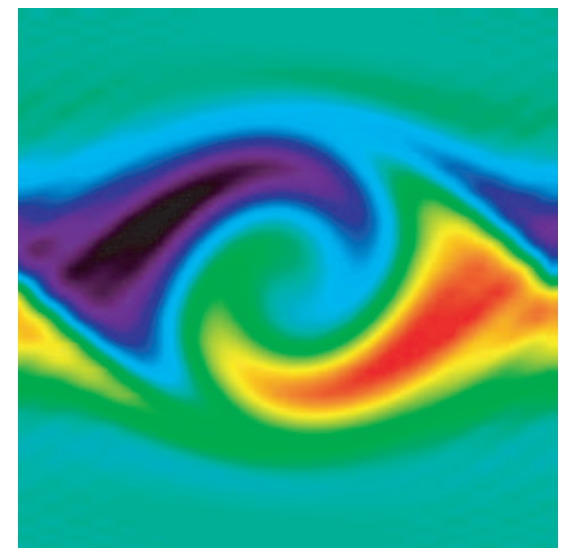

(b)

Figure 2. Magnetic field in the flow (2.4) with $R=500$. In (a) there is a Ponomarenko mode with $k=2.5$, and in (b) a cat's eye mode with $k=5$. In each case $B_{z}$ is plotted in the $(x, y)$-plane with positive field yellow/red and negative field blue/black.

In the case of Ekman instabilities, kinematic dynamo action was studied by Ponty et al. (2001). The magnetic fields may be taken proportional to exp(ikz), with a wavenumber $k$ along the axes of the cat's eyes. At a fixed, large magnetic Reynolds number $R$, magnetic field modes are unstable over a wide range of values of $k$. For moderate $k$, the unstable magnetic fields generally take the form of Ponomarenko modes. The field is confined to stream surfaces localised within each cat's eye and takes the form of spiralling tubes of field in three dimensions (figures $2 \mathrm{a}$ and $4 \mathrm{a}$ ). This type of dynamo action was first studied in a cylindrical geometry by Ponomarenko (1973) (see also the related model of Lortz (1968) and since by Gilbert (1988), Ruzmaikin et al. (1988), Solovyev (1985), Soward (1990) and Gilbert and Ponty (2000), amongst others). 
For larger $k$, the dominant mode takes a different form, of a magnetic field localised along the network of separatrices and hyperbolic stagnation points (figures $2 b, 4 b$ and 5). It appears to grow more strongly than the Ponomarenko modes at large $R$ and has a smaller spatial scale. A mode with this general form may be called a 'separatrix mode', as it localises on the network of separatrices and thrives on the exponential stretching at hyperbolic points in the network. We will take the term 'separatrix mode' to refer to any such mode that localises in this way at large $R$; however there can be different subfamilies depending on the topology of the separatrices.

One important subfamily is that of 'Roberts modes', which are found in the flow of Roberts (1972) and have been studied at large $R$ analytically in Soward (1987) and numerically in a related flow by Plunian et al. (1999). In these cases, there is a doubly periodic network of separatrices, bounding square cells, and a mean field on large scales possessing both $x$ and $y$ components. On the other hand, for the cat's eye geometry depicted in figure 1, the network of separatrices is periodic in just one direction, and defines a row of cat's eyes in the $(x, y)$-plane. We therefore adopt the term 'cat's eye mode', for a mode such as that in figure 2(b) where the field localises along a single row of cat's eyes; such modes constitute a subfamily of the separatrix mode family, distinct from the Robert's mode subfamily.

The aim of this article is to elucidate the properties of these cat's eye modes, and their links to other numerical and asymptotic studies. We consider kinematic dynamo action in a number of idealised flows; although these flows are not solutions of the Navier-Stokes equations with realistic boundary conditions, they allow a comprehensive exploration of the dynamo properties of general classes of flows, at modest computational cost. This is a well-used approach in dynamo theory; we may for example mention modelling of the Riga dynamo experiment (Gailitis et al. 2000) by the Ponomarenko (1973) dynamo, the Karlsruhe experiment (Stieglitz and Müller 2001) by the Roberts (1972) dynamo and the von Kármán sodium experiment (Bourgoin et al. 2002, Marie et al. 2002) by the Taylor-Green dynamo (Nore et al. 1997, Ponty et al. 2005).

The article is structured as follows. Section 2 studies a cat's eye mode present in the flow of figure 1, a plane layer bounded in the coordinate $y$, and elucidates the basic stretch-fold-shear mechanism by which the magnetic field can be amplified. For computational efficiency, and to compare with previous work, we then move to a doubly periodic geometry in section 3. Here, there are multiple and parallel rows of cat's eyes, separated by channels. We vary the wavenumber $k$, and $R$, to see the different types of modes that can be excited and their properties. For large-scale magnetic modes with $k \ll 1$, the $\alpha$-effect becomes applicable, as determined by Childress and Soward (1989), and we compare our results with this theory in section 4. Finally, section 5 offers a concluding discussion.

\section{Cat's eye modes in a plane layer}

We solve the induction equation, written in the form

$$
\frac{\partial \mathbf{B}}{\partial t}=\nabla \times(\mathbf{u} \times \mathbf{B})+\varepsilon \nabla^{2} \mathbf{B}, \quad \nabla \cdot \mathbf{B}=0,
$$


for a given flow $\mathbf{u}(x, y)$ having scale and turnover time of order unity. Here, $R=\varepsilon^{-1}$ is the magnetic Reynolds number, taken to be large, with $0<\varepsilon \ll 1$. We consider eigenmodes with wavenumber $k$,

$$
\mathbf{B}=\mathbf{b}(x, y) \mathrm{e}^{p t+\mathrm{i} k z}+\text { complex conjugate }
$$

The flow considered below is defined in the plane layer $-1 \leq y \leq 1$, and periodic in $x$, period $2 \pi$. We impose insulating boundary conditions on the magnetic field, which therefore matches to a potential field satisfying $\boldsymbol{\nabla} \times \mathbf{B}=0$ for $y>1$ or $y<-1$. In practise the choice of magnetic boundary condition is unimportant, as the modes we consider are (for large $R$ ) localised in the body of the fluid, away from the boundaries.

We consider a flow taking the incompressible form

$$
\mathbf{u}=\left(\frac{\partial \psi}{\partial y},-\frac{\partial \psi}{\partial x}, w\right), \quad \psi=\psi(x, y), \quad w=w(x, y)
$$

Our first example is the flow depicted in figure 1,

$$
\psi=-\frac{a y^{2}+b \cos x}{a+b \cos x}+\frac{b}{b-a}, \quad w=c \psi,
$$

with

$$
a=5, \quad b=-1, \quad c=1 .
$$

This flow is designed to be broadly similar to the flows seen for the Ekman instability (Hoffman et al. 1998, Ponty et al. 2001, 2003). However, note that in this flow the velocity $w$ is constant on stream lines of constant $\psi$. The quantities $\psi$ and $w$ are zero at the hyperbolic stagnation point $(\pi, 0)$ in the $(x, y)$-plane and on the corresponding separatrices, negative in the channels and positive in the cat's eyes, where the sense of the motion is anticlockwise. This feature that $w=w(\psi)$ is not the case in general for an Ekman instability, and this has implications for the way in which the field is folded at large $R$ in a flow such as (4). We will therefore consider a flow (12) below, which relaxes this condition.

A typical view of a cat's eye mode is shown in figure 2(b) from a run with $k=5$ and $R=500$. For contrast, figure 2(a) shows a Ponomarenko mode with $k=2.5$ and the same value of $R$. Plotted in each case is the $B_{z}$ component of field, in the $(x, y)$-plane, for a typical slice of constant $z$. Although the two pictures have some features in common, in particular blobs of each sign of field, in figure 2(b) the field has structure on the separatrices and hyperbolic points seen in the stream line pattern of figure 1 . The growth rate of magnetic modes is shown in figure 3 as a function of $k$ for $R=500$.

In figure 4, we show the magnetic field in three dimensions for these two cases. Again (a) shows the $k=2.5$ Ponomarenko mode, and (b) the $k=5.0$ cat's eye mode. In each case, isosurfaces of positive and negative $B_{z}$ field are shown as blue and red tubes, respectively. These show a similar configuration for the two modes; however in (b) an isosurface of constant $|\mathbf{B}|$ in green reveals that the tubes are linked to strong 


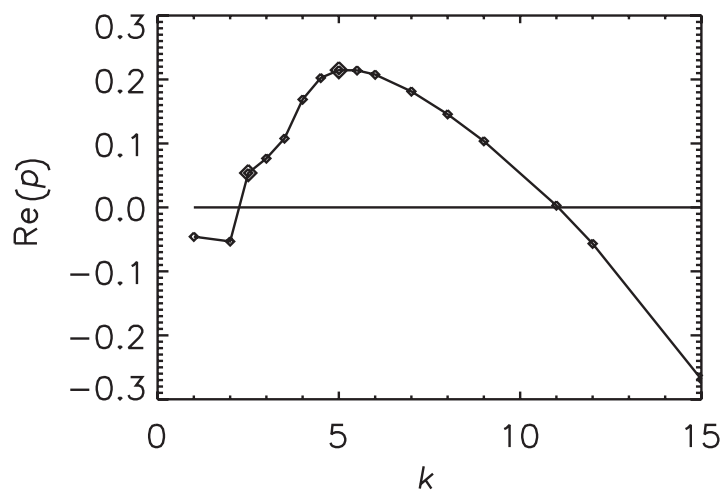

Figure 3. Growth rate $\operatorname{Re}(p)$ as a function of $k$ for $R=500$ and the flow (2.4) and (2.5). The points $k=2.5$ and $k=5$ are highlighted.

(a)

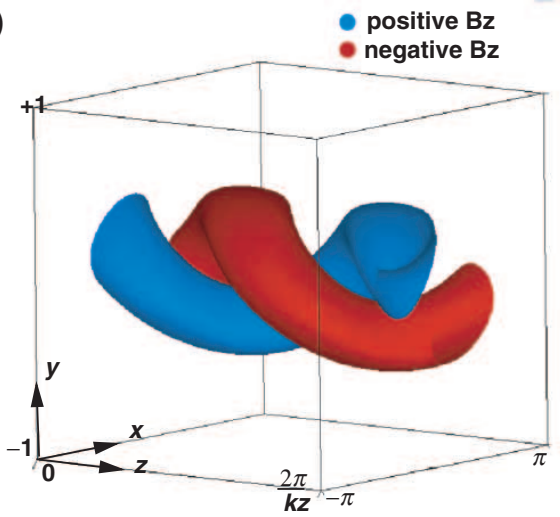

(b)

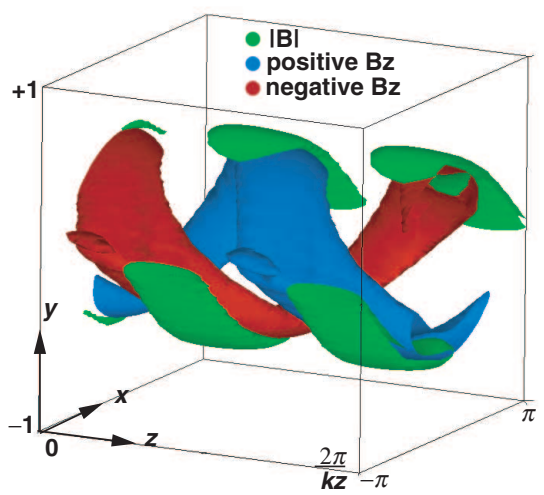

Figure 4. Magnetic field as in figure 2 but with plots of isosurfaces, $B_{z}=B_{z 0}$ (blue), $B_{z}=-B_{z 0}$ (red) and, in (b) only, $|\mathbf{B}|=B_{0}$ (green). In (a) $k=2.5$ and we have $B_{z 0}=0.5 \max B_{z}$ (with $\max B_{z} \simeq 0.6 \max |\mathbf{B}|$ ), and in (b) $k=5$ and the levels $B_{z 0}=0.65 \max B_{z}, B_{0}=0.65 \max |\mathbf{B}|$ are used (with $\max B_{z} \simeq 0.1 \max |\mathbf{B}|$ ).

sheets of field that lie on unstable manifolds of the hyperbolic stagnation points of the flow. The effect of the hyperbolic points appears to stretch the magnetic field into flux sheets along the unstable manifolds. This stretching, and compression of structure along the stable manifolds, is well known to intensify magnetic energy locally, and not magnetic flux, but flux amplification is the key ingredient required for any dynamo (fast or slow). At large $R$, it is natural to seek some folding mechanism that can achieve this. To show the flux sheets in greater detail, figure 5 protrays the isosurfaces of positive and negative $B_{x}$ (yellow, green) and $B_{y}$ (red, blue) field for the $k=5.0$ cat's eye mode only. The sheets now appear as yellow and green sheets intersecting the left- and right-hand sides of the box. However, the plot now reveals that the sheets are linked by $y$-directed fields. It appears that magnetic field from the tip of one sheet, say the yellow sheet, $B_{x}>0$, is carried around the edges of the cat's eyes, just inside, where it is turned to become positive $B_{y}$ field (red). Simultaneously, it is being translated in the $z$-direction so as to feed into the next sheet along, in this case the green sheet with $B_{x}<0$. 


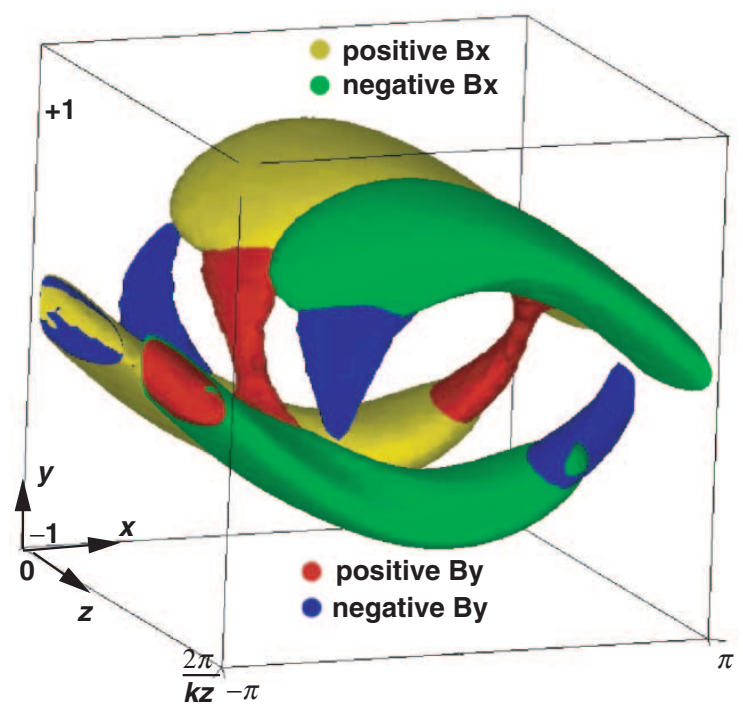

Figure 5. Magnetic field as in figure 4(b) with $k=5$, but with plots of isosurfaces, $B_{x}=B_{x 0}$ (yellow), $B_{x}=-B_{x 0}$ (green), $B_{y}=B_{y 0}$ (red), and $B_{y}=-B_{y 0}$ (blue). Here $B_{x 0}=0.25 \max B_{x}$ and $B_{y 0}=0.28 \max B_{y}$, with $B_{x 0} \simeq \max |\mathbf{B}|$ and $B_{y 0} \simeq 0.25 \max |\mathbf{B}|$.

(a)

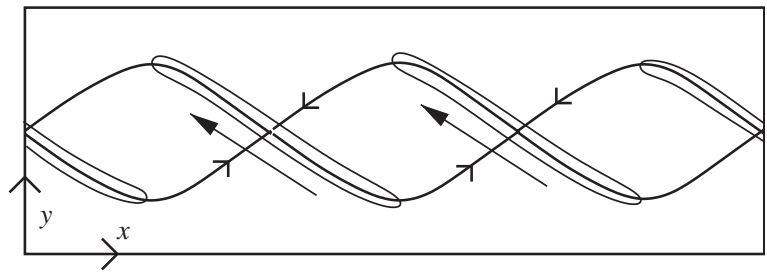

(b)

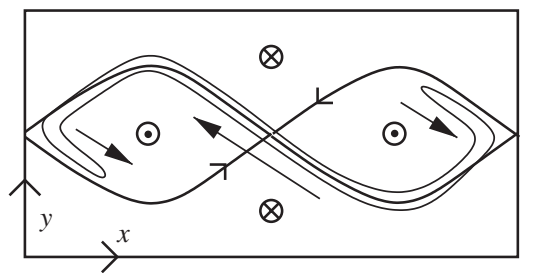

Figure 6. Schematic mechanism for a cat's eye mode, shown in the $(x, y)$-plane and described in the text.

This suggests a possible mechanism for the dynamo, depicted schematically in figure 6, and simply a variant of the well-known stretch-fold-shear mechanism (Bayly and Childress 1988) acting in a steady flow (Soward 1987). In figure 6(a) sheets of field are compressed at the hyperbolic stagnation points, and extend along their unstable manifolds. Heavy lines show the flow field and light lines/arrows show the magnetic field. In figure 6(b), we examine a single sheet, which diffuses inside the cat's eye and is carried around the eye, leading to a reversal of sign. Simultaneously, the $z$-component of the flow carries these reversed fields in the 
$z$-direction so that they reinforce a sheet with the opposite sign, that lies at a distance $\pi / k$ in the $z$-direction. Note that the fields depicted in this figure are not divergence free, but this is because we are sketching the $B_{x}$ and $B_{y}$ components only, and not trying to draw the $B_{z}$ component that can ensure that $\nabla \cdot \mathbf{B}=0$ is satisfied.

\section{Cat's eye modes in periodic geometry}

To understand better, the properties of cat's eye modes and how they fit in with other classes of dynamo modes, we move from the plane layer geometry to flows that are doubly periodic in the $(x, y)$-plane. This class of flows has been widely studied in terms of dynamo action since Roberts (1972). We consider a cat's eye flow with

$$
\psi=A \cos x-B \cos y, \quad w=\psi
$$

that is,

$$
\mathbf{u}=A(0, \sin x, \cos x)+B(\sin y, 0,-\cos y) .
$$

This is a member of the family of ABC flows (with $C=0$ ), defined by Beltrami (1920), Arnold (1965) and Childress (1967). Dynamo action in ABC flows was first studied by Roberts (1972), Arnold and Korkina (1983), and Galloway and Frisch (1986). The flow (7) is defined in an equivalent form by Childress and Soward (1989), who study dynamo action for low $k$, as we will discuss shortly. Provided $|A| \neq|B|$ the flow has a pattern of cat's eyes in the $(x, y)$-plane, separated by channels. As an example, we use the parameter values

$$
A=\sqrt{3 / 5}, \quad B=2 A,
$$

and the flow is depicted in figure 7.

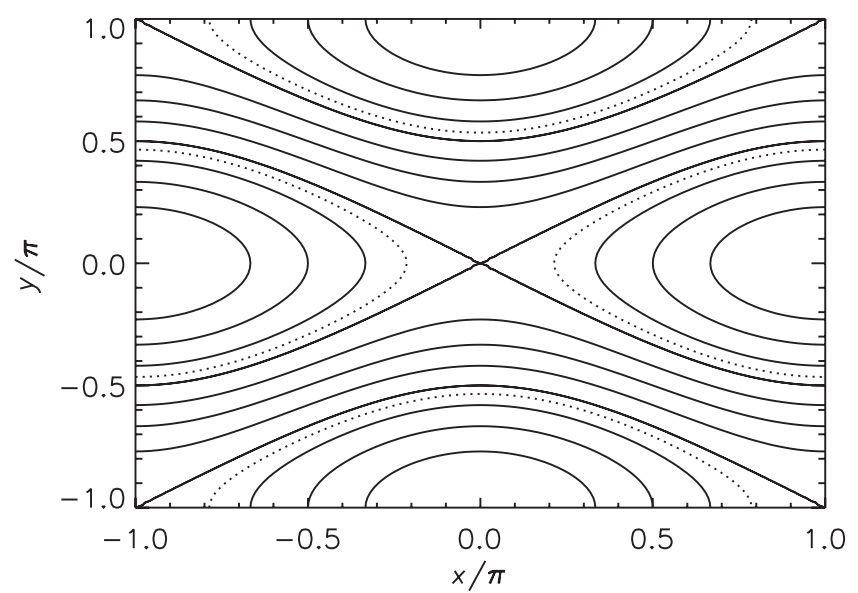

Figure 7. Stream lines of constant $\psi(x, y)$ are shown for the flow (3.1), (3.3) with $|\psi|=\psi_{\mathrm{c}}$ dotted. 

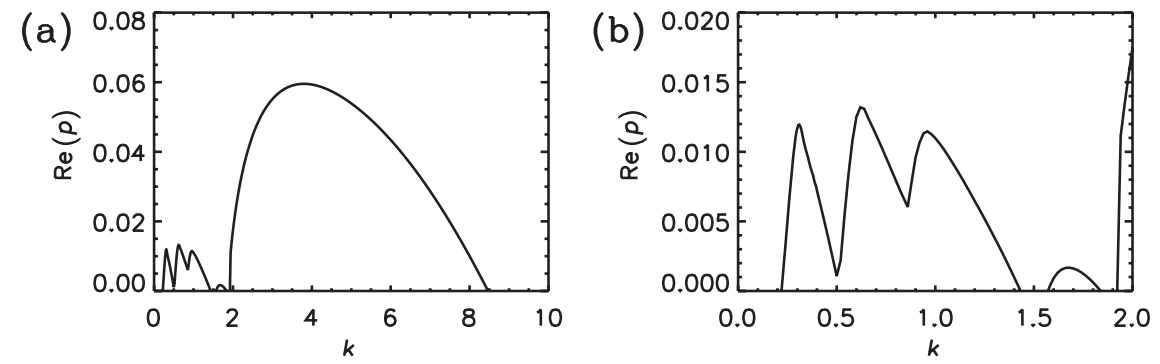

Figure 8. Magnetic field growth rate $\operatorname{Re}(p)$ as a function of $k$ for $R=1000$ and the flow (3.2) and (3.3); (b) is simply a magnified view of the bottom left corner of (a).

(a)

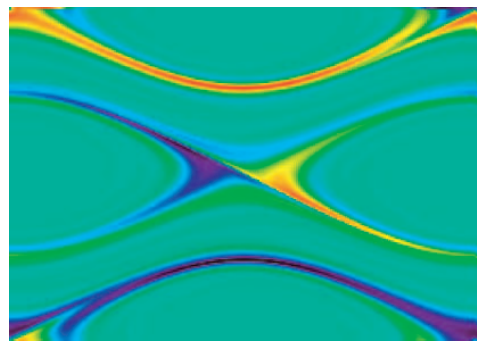

(c)

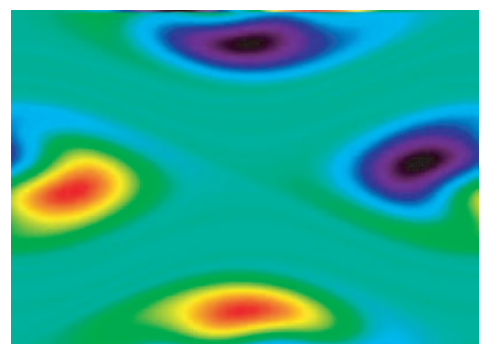

(b)

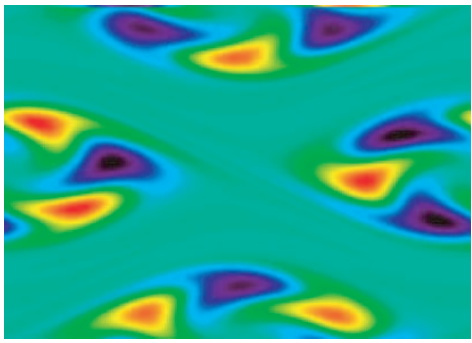

(d)

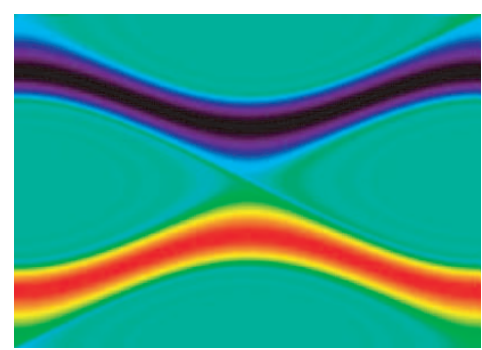

Figure 9. Magnetic field structure for $R=1000$ and (a) $k=3.8$ (cat's eye mode), (b) $k=1.0$ (Ponomarenko mode, $m=3$ ), (c) $k=0.3$ (Ponomarenko mode, $m=1$ ), and (d) $k=0.001$ ( $\alpha$-effect mode). In each case $B_{z}$ is plotted in the $(x, y)$-plane with positive field yellow/red and negative field blue/black.

We have examined the growth rates of magnetic fields for various values of $R$ and ranges of $k$. Figure 8 shows the growth rate $\operatorname{Re}(p)$ plotted against $k$ for $R=1000$. There are a number of peaks in the curve. Corresponding magnetic fields for certain $k$-values are depicted in figure 9 , which shows $B_{z}$ in the $(x, y)$-plane, and in figure 10 , which gives the $z$-averaged magnetic energy $|\mathbf{b}|^{2}$, from (2). Note that the fields in figure 9 depend on the slice in $z$ chosen, whereas those in figure 10 are independent of $z$.

The most prominent feature of figure 8 is the peak of maximum growth rate at $k \simeq 3.8$, for which the corresponding magnetic field is shown in figures 9(a) and 10(a). This is clearly the cat's eye mode which we have already seen in the plane layer simulation; the magnetic field is localised along individual rows of cat's eyes, and vanishes within the channels and within the cat's eyes. 
(a)

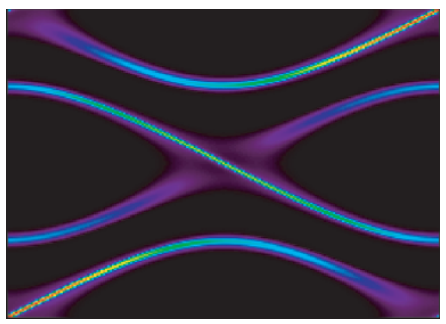

(c)

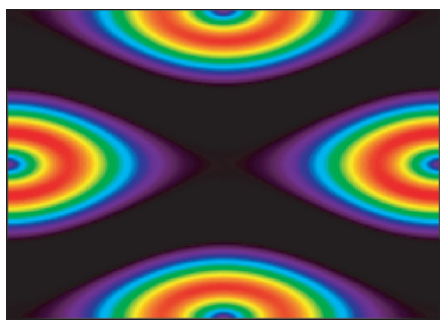

(b)

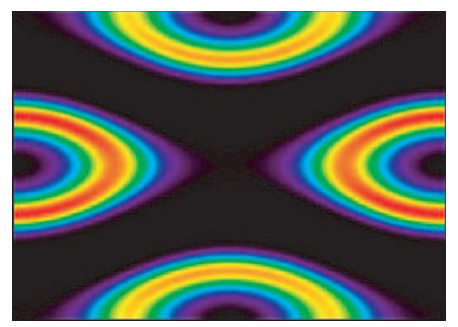

(d)

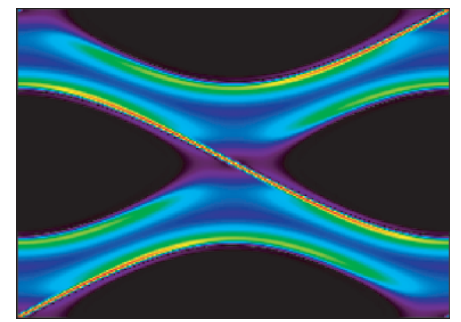

Figure 10. Magnetic field structure as in figure 9 but with the $z$-averaged energy density $|\mathbf{b}|^{2}$ plotted in each case and black corresponding to zero energy density, red to maximal density.

Reducing $k$, there is then a series of peaks in the range $0<k<2$. These appear to be Ponomarenko modes, and two with $m=3$ and $m=1$ are depicted in figures $9(\mathrm{~b}, \mathrm{c})$ and $10(\mathrm{~b}, \mathrm{c})$. Ponomarenko modes localise on stream surfaces of constant $\psi$ in the limit of large $R$, and vary as $\exp (\mathrm{i} m \vartheta+\mathrm{i} k z)$, where $\vartheta$ is an angle coordinate on the closed stream line in the $(x, y)$-plane defining the stream surface. The stream surface itself is given by a resonance condition $m \Omega^{\prime}(\psi)+k w^{\prime}(\psi)=0$, where $\Omega(\psi)$ is the angular velocity around the stream line and the prime denotes a derivative. This theory of smooth Ponomarenko dynamos at large $R$ was established for cylindrical geometry by Gilbert (1988) and Ruzmaikin et al. (1988), and generalised by Soward (1990) and Gilbert and Ponty (2000). A given stream surface can only support a growing mode provided the geometrical condition

$$
\left|\frac{\Omega^{\prime \prime}(\psi)}{\Omega^{\prime}(\psi)}-\frac{w^{\prime \prime}(\psi)}{w^{\prime}(\psi)}\right|<4 \frac{\left|\alpha_{m}\right|}{\gamma}
$$

is met, using the notation of Gilbert and Ponty (2000) which should be referred to for definitions of $\alpha_{m}$ and $\gamma$. Checking this criterion numerically for the cat's eye flow with the parameters (3.3) indicates that the Ponomarenko dynamo action can only occur on stream surfaces with $|\psi|>\psi_{\mathrm{c}} \simeq 0.943$. The stream lines $\psi= \pm \psi_{\mathrm{c}}$ are shown dotted in figure 7. It may be seen that the Ponomarenko modes in figures $9(\mathrm{~b}, \mathrm{c})$ and $10(\mathrm{~b}, \mathrm{c})$ are localising in the cat's eyes inside those stream surfaces, and the cat's eye mode in figures 9 (a) and 10 (a) in the region $|\psi|<\psi_{\mathrm{c}}$ containing the separatrices.

This confirms that the cat's eye modes seen are distinct from the Ponomarenko modes. Another important feature is the scaling of growth rates as a function of $k$ at large $R$. Whereas the fastest growing Ponomarenko mode has the scalings

$$
m, k=\mathrm{O}\left(R^{1 / 3}\right), \quad \operatorname{Re}(p)=\mathrm{O}\left(R^{-1 / 3}\right),
$$


and spatial scale across stream surfaces of order $R^{-1 / 3}$ (e.g. Gilbert and Ponty 2000), the cat's eye mode has faster growth rates and smaller scales. Figure 11 shows the wave number and growth rate for the fastest growing cat's eye mode. There appears to be a scaling of

$$
k=\mathrm{o}\left(R^{1 / 2}\right), \quad \operatorname{Re}(p)=\mathrm{o}(1)
$$

where the o symbol has the usual meaning, but here we use it to indicate a dependence that is slower than a power law in $R$. We generally expect this type of scaling: the growth rate must go to zero for large $R$ as a steady, smooth two-dimensional flow is necessarily a slow dynamo (Klapper and Young 1995). On the other hand, the analytical study of Roberts modes shows growth rates going to zero extremely slowly (Soward, 1987), as $\mathrm{O}(\log (\log R) / \log R)$, and $k$ scaling as $\mathrm{O}\left((R / \log R)^{1 / 2}\right)$. We appear to have a broadly similar situation here, as far as we can tell numerically. In any case, the scaling observed is plainly distinct from that in (10) for Ponomarenko modes.

For reasons discussed below (2.5), let us now replace (6) by

$$
\psi=A \cos x-B \cos y, \quad w=A \cos x-B \cos (y-\phi),
$$

where $\phi$ is a fixed angle. For a general value of $\phi$ the spatial pattern of the vertical velocity $w$ is shifted in the $y$-direction and is no longer a function of $\psi$. Figure 12 shows growth rate against $k$ for $R=1000, \phi=\pi / 36$ and $\phi=\pi / 12$. We see that the
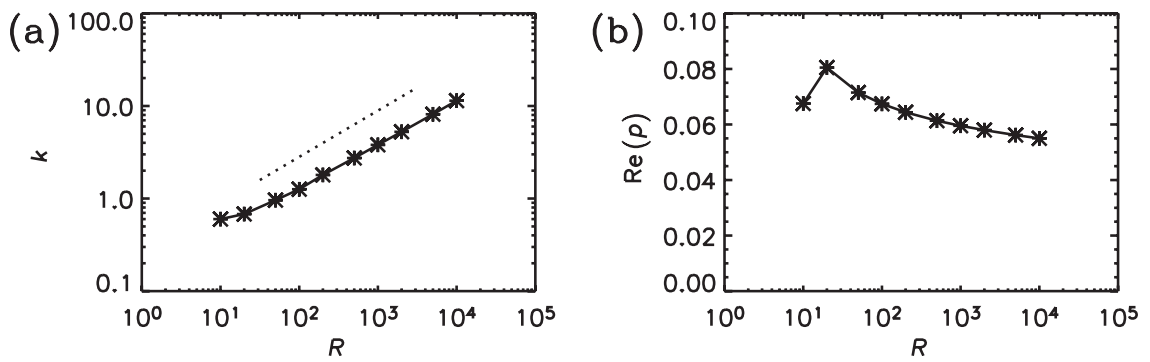

Figure 11. In (a) the wave number $k$ and (b) the growth rate $\operatorname{Re}(p)$, for the fastest growing cat's eye mode, are plotted against $R$. The dotted line in (a) shows the scaling $k \propto R^{1 / 2}$.
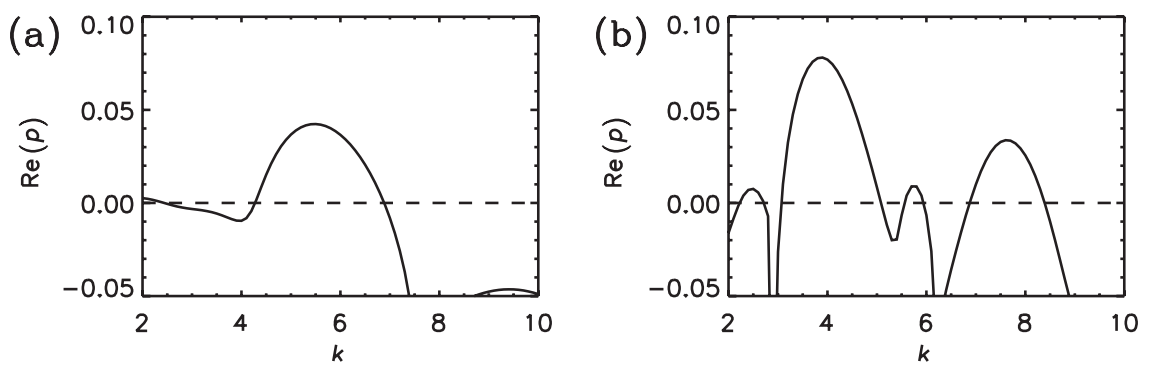

Figure 12. Magnetic field growth rate $\operatorname{Re}(p)$ as a function of $k$ for $R=1000$ and flow (3.7) with (a) $\phi=\pi / 36$ and (b) $\phi=\pi / 12$. 
very broad peak evident in figure 8 first of all narrows as $\phi$ is increased from zero, and growth rates are reduced. As $\phi$ is increased further, the peak breaks up and the growth rate now has an oscillatory form as a function of $k$.

To see why these oscillations arise, consider first the case when $w=w(\psi)(\phi=0)$ and work in a frame in which $w=0$ at the hyperbolic points. Then $w=0$ along the separatrices also, and so field has to diffuse off the separatrix in order to be carried in the $z$-direction and reinforce neighbouring bands of field (as shown in figure 5). Now when $w \neq w(\psi)$, and in the frame in which $w=0$ at the hyperbolic points, there will be a net displacement, say $\Delta$, as a particle moves around very close to the separatrices in a circuit of one cat's eye. Whether or not this displacement is favourable to the feeding of a folded sheet of field into neighbouring sheets will depend on the wavenumber $k$, and we expect oscillations as $k$ is varied, as observed. This may also explain the complex variation of growth rate against $k$ that emerges in the study of dynamo action in Ekman layer instabilities, for which $w$ is not generally a function of $\psi$; see figure 5 of Ponty et al. (2001).

Finally, we consider how the cat's eye modes that we have found, with $A \neq B$, are linked to those for the Roberts (1972) flow, which has $A=B$. One convenient way to link the two flows is to consider the family parameterised by an angle $\xi$, with

$$
A=\sqrt{3} \sin \xi, \quad B=\sqrt{3} \cos \xi
$$

This family has fixed kinetic energy (with $A^{2}+B^{2}=3$ ) and varies from the Roberts flow with $\xi=45^{\circ}$ to our flow (3.3) given by $\tan \xi=1 / 2$, and to unidirectional Kolmogorov flow when $\xi=0$. Figure 13 shows growth rate $\operatorname{Re}(p)$ plotted against $k$ for $\xi$ decreasing from $45^{\circ}$ to $42^{\circ}$. Notable is the rapid disappearance of the Roberts mode for $\xi=45^{\circ}$, to be replaced by the distinct cat's eye mode, which has lower growth rates. This is not so surprising, as the field (especially for large $k$ ) becomes sensitive to the presence of channels when the distance across the channel is of order $R^{-1 / 2}$ and this occurs when $|A-B|$ or $45^{\circ}-\xi$ are of this order. The picture changes little as $\xi$ is reduced further to our flow with $\tan \xi=1 / 2$.

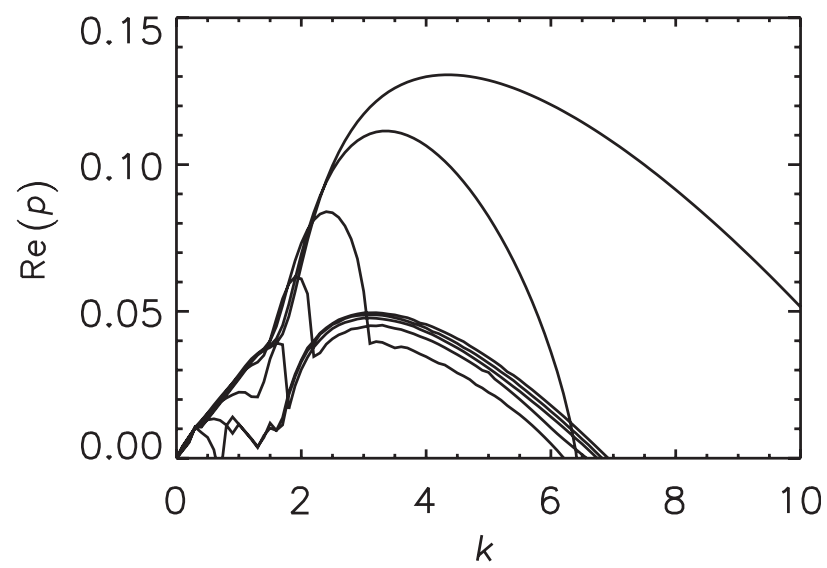

Figure 13. Growth rate $\operatorname{Re}(p)$ as a function of $k$ for $R=1000$ and $\xi=45^{\circ}, 44.5^{\circ}, 44^{\circ}, 43.5^{\circ}, 43^{\circ}, 42.5^{\circ}$ and $42^{\circ}$, reading the curves from top right to bottom left. 


\section{Modes for $k \ll 1$ governed by the $\alpha$-effect}

The cat's eye flow (6) has been studied analytically for magnetic fields possessing a large scale in the $z$-direction, $0<k \ll 1$, in the limit of large $R$, by Childress and Soward (1989). In this case, an $\alpha$-effect description becomes appropriate. A constant magnetic field is imposed (i.e. with $k=0$ ) initially, and the field is allowed to evolve to a steady state. The $\alpha$-effect matrix $\alpha$ is then determined by measuring the induced emf $\overline{\mathbf{u} \times \mathbf{B}}=\alpha \overline{\mathbf{B}}$ in the usual way, where the overline denotes an average over the $(x, y)$-plane. In view of the symmetry of the flow the $\alpha$-matrix is diagonal, with entries $\alpha_{1}$ and $\alpha_{2}$, say. Then a mean field that varies slowly in $z$ satisfies

$$
\frac{\partial \bar{B}_{1}}{\partial t}=-\frac{\partial}{\partial z}\left(\alpha_{2} \bar{B}_{2}\right), \quad \frac{\partial \bar{B}_{2}}{\partial t}=\frac{\partial}{\partial z}\left(\alpha_{1} \bar{B}_{1}\right)
$$

and a mode depending as $\exp (\mathrm{i} k z)$ has growth rate

$$
p \simeq \pm k \sqrt{\alpha_{1} \alpha_{2}}
$$

This may be viewed as the leading term in a Taylor expansion for small $k$. The next terms in the expansion would contribute an $\mathrm{O}\left(k^{2}\right)$ term that may be identified with an eddy diffusion term.

According to the theory of Childress and Soward (1989), the $\alpha_{j}$ components scale as

$$
\alpha_{1}=\mathrm{O}\left(R^{-3 / 2}\right), \quad \alpha_{2}=C_{1} R
$$

The large-scale growing magnetic field is a helical wave, but with $\left\langle\bar{B}_{1}^{2}\right\rangle_{z} /\left\langle\bar{B}_{2}^{2}\right\rangle_{z}=\left|\alpha_{2} / \alpha_{1}\right|$. Thus, in the limit of large $R$, the mean $\bar{B}_{1}$ field becomes the dominant component. Field is expelled from the cat's eyes, and the mode is depicted in figures $9(\mathrm{~d})$ and $10(\mathrm{~d})$. Note that from figure 10(d), the strongest fields lie on the separatrices joining the hyperbolic points, as for the cat's eye mode, but there are additionally fields within the channels, and these are crucial for the mode to communicate across channels and possess a large-scale field.

To confirm the scalings in (16), figure 14 shows the $\alpha_{j}$ components measured numerically, and there is good agreement. Note that in (b), the constant $C_{1}$ is included,

(a)

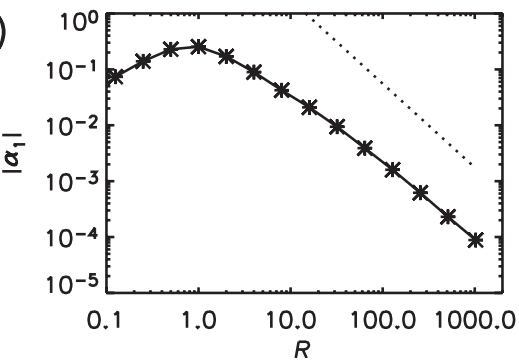

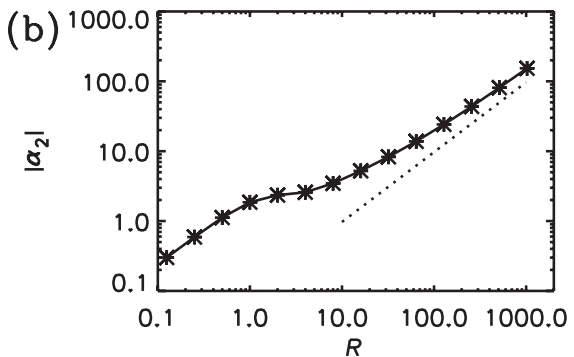

Figure 14. Components (a) $\left|\alpha_{1}\right|$ and (b) $\left|\alpha_{2}\right|$, measured numerically and plotted against $R$ for the cat's eye flow with the parameters (3.3). Dotted lines show the theoretical results in (4.3). In (a) only the scaling law is shown, but in (b) the constant $C_{1}$ is given from Childress and Soward (1989). 
being defined precisely below; this is given by the theory of Childress and Soward (1989), valid in the limit $A \simeq B$, or $\delta \ll 1$, with $\delta$ defined below in (20). Given that here $\delta=1 / 3$, the agreement is fair.

The $\alpha$-effect gives a window of dynamo action that exists only for low $k$, in fact in a range $0<k \leq \mathrm{O}\left(R^{-1}\right)$ that becomes narrow as $R$ increases. This is explained by Childress and Soward (1989): an $\alpha$-effect dynamo requires large-scale fields in $x$ - and $y$-directions with components within the channels as seen. However, the vertical shear within the channels is very effective at destroying such fields if they vary in $z$, unless they vary only on a very large, $\mathrm{O}(R)$, length scale. The window of dynamo action for very low $k$ is not visible in figure 8 and indeed it is time-consuming to determine it accurately at large $R$. Instead figure 15 shows results for $R=32,64$ and 128, for low values of $k$. The dotted lines give the results from the numerical computations of $\alpha_{1}$ and $\alpha_{2}$ in figure 14, substituted into the formula (15); there is good agreement with theory, and we can see clearly how the window of dynamo action controlled by the $\alpha$-effect shrinks as $R$ is increased.

We have undertaken further runs to check the boundary layer calculations of Childress and Soward (1989). They find it convenient to write the flow as

$$
\psi^{\prime}=\sin x^{\prime} \sin y^{\prime}+\delta \cos x^{\prime} \cos y^{\prime}, \quad w^{\prime}=\sqrt{2} \psi^{\prime} .
$$

Explicitly the transformation required to obtain this form is from $(x, y, z, t)$ to $\left(x^{\prime}, y^{\prime}, z^{\prime}, t^{\prime}\right)$ with

$$
L\left(x^{\prime}+\pi\right)=2^{-1 / 2}(x-y), \quad L y^{\prime}=2^{-1 / 2}(x+y), \quad L z^{\prime}=z, \quad t^{\prime}=t U / L,
$$

and other quantities become

$$
R^{\prime}=R L U, \quad k^{\prime}=k L, \quad p^{\prime}=p L / U, \quad \alpha_{j}^{\prime}=\alpha_{j} / U
$$

where

$$
U=2^{-1 / 2}(A+B), \quad L=\sqrt{2}, \quad \delta=(B-A) /(B+A) .
$$

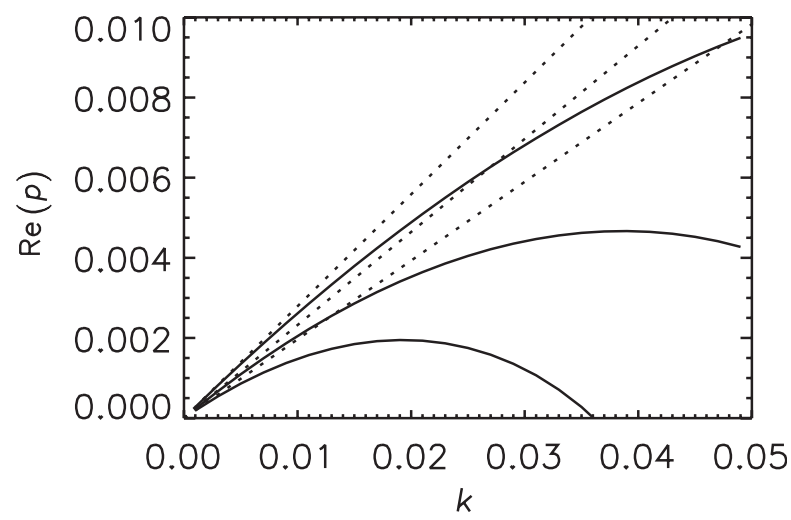

Figure 15. Magnetic field growth rate $\operatorname{Re}(p)$ as a function of $k$, for $k$ small and $R=32,64$ and 128 (reading down the solid curves). The dotted curves give the low- $k$ prediction from the $\alpha$-effect calculation. 
Table 1. Comparison with table 1 of Childress and Soward (1989). Here $\beta=\delta R^{\prime 1 / 2}$. Columns 3-5 give results for $R^{1 / 2} \alpha^{(+)}$and columns 6-8 give those for $R^{\prime 1 / 2} \alpha^{(-)}$. The labels are 'sim' for results computed numerically using a two-dimensional simulation for the value of $R^{\prime}$ given, 'bl' for the numerical solution of the boundary layer equations (valid in the limit $R^{\prime} \rightarrow \infty$ ) taken from Childress and Soward (1989), and 'asy' for their asymptotic approximation valid for large $R^{\prime}$ and large $\beta$.

\begin{tabular}{|c|c|c|c|c|c|c|c|}
\hline$\beta$ & $R^{\prime}$ & $R^{\prime 1 / 2} \alpha^{(+)}: \operatorname{sim}$ & $\mathrm{bl}$ & asy & $R^{\prime 1 / 2} \alpha^{(-)}: \operatorname{sim}$ & $\mathrm{bl}$ & asy \\
\hline 0.5 & 25 & 0.2214 & & & 1.3792 & & \\
\hline 0.5 & 64 & 0.2227 & & & 1.3805 & & \\
\hline 0.5 & 100 & 0.2232 & & & 1.3812 & & \\
\hline 0.5 & $\infty$ & & 0.2246 & 0.2248 & & 1.383 & 1.320 \\
\hline 1.0 & 64 & 0.1172 & & & 3.3582 & & \\
\hline 1.0 & 100 & 0.1177 & & & 3.3646 & & \\
\hline 1.0 & 144 & 0.1179 & & & 3.3680 & & \\
\hline 1.0 & $\infty$ & & 0.1186 & 0.1250 & & 3.377 & 3.455 \\
\hline
\end{tabular}

The $\alpha$-tensor has eigenvalues $\alpha_{1}^{\prime}$ and $\alpha_{2}^{\prime}$ but now takes the form

$$
\alpha^{\prime}=\frac{1}{2}\left(\begin{array}{ll}
\alpha_{1}^{\prime}+\alpha_{2}^{\prime} & \alpha_{1}^{\prime}-\alpha_{2}^{\prime} \\
\alpha_{1}^{\prime}-\alpha_{2}^{\prime} & \alpha_{1}^{\prime}+\alpha_{2}^{\prime}
\end{array}\right) .
$$

Note that $\alpha_{1}^{\prime}=-L \alpha^{(+)}$and $\alpha_{2}^{\prime}=-L \alpha^{(-)}$, in the notation of Childress and Soward (1989) (see their equation (1.20)). The scaling $\alpha^{(-)}=(2 / 3) \delta^{3} R^{\prime}$ given in their equation (3.23) for $\delta \ll 1$, then becomes $\alpha_{2}=C_{1} R$ with $C_{1}=-(2 / 3) L^{2} U^{2} \delta^{3}$, and this is what used in figure $14(\mathrm{~b})$.

Using the Childress and Soward (1989) coordinate system and related quantities, table 1 gives results for the $\alpha$-effect computed in our simulations for comparison. There is further explanation in the table caption. They consider a limit in which the channel width goes to zero as $R^{\prime} \rightarrow \infty$, keeping the parameter $\beta=\delta R^{1 / 2}$ constant. There is good agreement, and our simulations for moderate $R^{\prime}$ confirm their boundary layer calculations, valid for large $R^{\prime}$ and their asymptotic theory, valid for large $R^{\prime}$ and large $\beta$.

\section{Discussion}

We have discussed dynamo action in flows with rows of cat's eyes and identified a family of cat's eye modes. The cleanest picture came out of studying the doubly periodic ABC flow (with $C=0, A<B$ ). In this case, there is a clear hierarchy of modes. For very low $k$, we have the modes identified by Childress and Soward (1989) which can be described by an $\alpha$-effect. These may be thought of as global modes, in that they involve a $z$-dependent mean field with both an $x$ - and a $y$-component. Such fields cannot exist in a bounded plane layer (under perfectly conducting or insulating boundary conditions) and so this mode is special to the doubly periodic geometry. The mode also requires communication between rows of cat's eyes, across the channels, and this means that it only exists as a growing mode in a very small range of $k \leq \mathrm{O}\left(R^{-1}\right)$.

From these global modes, the next set in our hierarchy is the cat's eyes family that we have explored. In this case, the field lives along a single row of cat's eyes, but does not communicate across the channels. Such modes are equally at home in the doubly 
periodic and singly periodic plane layer geometries. They represent very efficient amplifiers of magnetic fields, with growth rate falling off very slowly with $R$; however their scale is small, with $k=\mathrm{o}\left(R^{1 / 2}\right)$, as far as we can tell numerically. Finally we have the Ponomarenko modes, which are the most localised, and can live in a single cat's eye, or a single convective cell. Their properties are well understood; they grow slowly by an $\alpha \omega$ mechanism available on curved stream-surfaces, and the magnetic fields have less fine-scale structure.

This raises the natural question of whether there are other classes of dynamo modes in two-dimensional (or axisymmetric) flows at large $R$ (and isolated from any boundaries). One possibility is that the channels between cat's eyes may themselves support growing magnetic fields. There are two possible types of channel available, those in which each streamline is periodic (necessarily the case in the plane layer geometry) and those for which stream lines are aperiodic (only possible in doubly periodic geometry). In the periodic case, we have not observed growing modes, but have no reason to believe that they should not exist for sufficiently general vertical velocities. In the aperiodic case, an individual streamline will visit every part of the channel along its length and come arbitrarily close to hyperbolic points and their separatrices. In this case, the alpha effect has been studied by Soward and Childress (1990), but there has been no study of dynamo action for finite $k$; here the sheets of magnetic field from the cat's eyes should accumulate in the channels as $R$ increases, and this family of modes would be worth studying.

The families we have considered in this article are only really defined in the sense of their asymptotic structure in the limit $R \rightarrow \infty$, and all three classes are seemingly distinct in this limit, having very different scalings for their growth rates and preferred wavenumbers $k$. For the ABC cat's eye flow, there is a gap between stream surfaces $|\psi|>\psi_{\mathrm{c}}$ which can support growing Ponomarenko modes, and the separatrix, which supports the cat's eye mode. This gap in physical space between the two classes of modes was identified by Soward (1990), and appears to rely on the property $w=w(\psi)$ of the vertical flow. For the cat's eyes flow, a streamline close to the separatrix $\psi=A-B$ has period given approximately by

$$
T(\psi) \equiv \frac{2 \pi}{\Omega(\psi)} \simeq \frac{2}{\sqrt{A B}} \log \left[\frac{32 A B}{\mu(B-A)}\right]
$$

where $\psi=A-B-\mu$ with $0<\mu \ll 1$. Thus $\Omega^{\prime \prime}(\psi) / \Omega^{\prime}(\psi)$ diverges as $\mu \rightarrow 0$, whereas if $w=w(\psi)$ depends smoothly on $\psi$, then $w^{\prime \prime}(\psi) / w^{\prime}(\psi)$ remains finite. With further analysis from Gilbert and Ponty (2000) it is found that

$$
\alpha_{m} / \gamma \simeq \Omega^{\prime} / 2 \Omega
$$

independently of the details of the motion along the separatrix. This means that the inequality (3.4) is necessarily violated as $\mu \rightarrow 0$ (Soward 1990), and Ponomarenko modes are isolated from the separatrices.

However, if we now write down some general $w(x, y)$, divorced from $\psi$ (that is, not any particular function of $\psi$ ), the situation changes. In a frame in which the vertical velocity is zero at the hyperbolic points, the mean velocity in the $z$-direction will be approximately $\bar{w}(\psi)=\Delta / T(\psi)$ where $\Delta$ is the total $z$-displacement of a particle on 
one traversal of the two separatrices defining the eye. When (9) is generalised to account for non-constant vertical velocity the left-hand side becomes $\mid \Omega^{\prime \prime}(\psi) / \Omega^{\prime}(\psi)-$ $\bar{w}^{\prime \prime}(\psi) / \bar{w}^{\prime}(\psi) \mid$, but this vanishes at leading order because now $\bar{w} \propto \Omega$.

In short, a flow with $w=w(\psi)$ has certain properties not shared by flows with general $w$, and further investigation of the case of general $w$ would be of interest. In particular, generally is there a gap between Ponomarenko modes and separatrix modes, or not, and does this have implications for the modes that are actually realised in a kinematic dynamo? We leave this as a topic for future study, but note that in this case another $\alpha$-effect, arising from vertical motion, has now to be included in the right-hand side of the generalisation of (9), giving additional complications.

It would also be interesting to study the cat's eye modes found analytically, and develop a general theory for their growth rates. Here, the aim would be to work with a quite general stream function $\psi$ having cat's eye topology, and a general vertical velocity $w$. The magnetic fields would have behaviour at the hyperbolic points matched with that along the separatrices, in a fusion of the studies Soward (1987), Childress and Soward (1989), and Gilbert and Ponty (2000). Finally, it is of course valuable to study the saturation of such growing magnetic fields, and this is the current work in progress for dynamos based on the Ekman instability (Zhang and Gilbert 2005).

\section{Acknowledgements}

This study was completed during the programme 'Magnetohyodrodynamics of Stellar Interiors' (Autumn 2004) at the Isaac Newton Institute, Cambridge. We are grateful to the organisers and director for inviting us to participate. We also thank Steve Childress, David Hughes, Franck Plunian, Andrew Soward and Steve Tobias for helpful discussions, and the referees for useful comments.

$\mathrm{AG} / \mathrm{YP}$ are grateful to CNRS/Royal Society for funding a programme of visits between Nice and Exeter and to the Leverhulme Trust for support. AC is pleased to acknowledge funding through the EPSRC and the University of Leeds. YP thanks the CNRS Dynamo GdR, and INSU/PNST and INSU/PCMI programmes for support. Computer time was provided by IDRIS (CNRS).

\section{References}

Arnold, V.I., Sur la topologie des écoulements stationnaires des fluides parfaits. C. R. Acad. Sci. Paris, 1965, 261, 17-20.

Arnold, V.I. and Korkina, E., The growth of a magnetic field in a three-dimensional steady incompressible flow. Vest. Mosk. Un. Ta. Ser. 1, Matem. Mekh., 1983, 43-46.

Bayly, B.J. and Childress, S., Construction of fast dynamos using unsteady flows and maps in three dimensions. Geophys. Astrophys. Fluid Dynam., 1988, 44, 211-240.

Beltrami, E., Opera Matematiche, Vol. 4, p. 304, Milan: Faculty of Science of the University of Rome, 1920.

Bourgoin, M., Marie, L., Petrelis, F., Gasquet, C., Guigon A., Luciani, J.B., Moulin, M., Namer, F., Burguete, J., Chiffaudel, A., Daviaud, F., Fauve, S., Odier, P. and Pinton, J.-F., Magnetohydrodynamics measurements in the von Kármán sodium experiment. Phys. Fluids, 2002, 14, 3046-3058.

Childress, S., Construction of steady-state hydromagnetic dynamos. I. Spatially periodic fields. 1967, Report MF-53, Courant Institute of Mathematical Sciences.

Childress, S. and Soward, A.M., Scalar transport and alpha-effect for a family of cat's-eye flows. J. Fluid Mech., 1989, 205, 99-133. 
Gailitis, A.K., Lielausis, O.A., Dement'ev, S., Platacis, E., Cifersons, A., Gerbeth, G., Gundrum, T., Stefani, F., Christen, M., Hänel, H. and Will, G., Detection of a flow induced magnetic field eigenmode in the Riga dynamo facility. Phys. Rev. Lett., 2000, 84, 4365-4368.

Galloway, D.J. and Frisch, U., Dynamo action in a family of flows with chaotic streamlines. Geophys. Astrophys. Fluid Dynam., 1986, 36, 53-83.

Gilbert, A.D., Fast dynamo action in the Ponomarenko dynamo. Geophys. Astrophys. Fluid Dynam., 1988, 44, 214-258.

Gilbert, A.D. and Ponty, Y., Dynamos on stream surfaces of a highly conducting fluid. Geophys. Astrophys. Fluid Dyn., 2000, 93, 55-95.

Hoffman, N., Busse, F.H. and Chen, W.-L., Transition to complex flows in the Ekman-Couette layer. J. Fluid Mech., 1998, 366, 311-331.

Klapper, I. and Young, L.S., Bounds on the fast dynamo growth rate involving topological entropy. Comm. Math. Phys., 1995, 173, 623-646.

Lortz, D., Exact solutions of the hydromagnetic dynamo problem. Plasma Phys., 1968, 10, 967-972.

Marie, L., Petrelis, F., Bourgoin, M., Burguete, J., Chiffaudel, A., Daviaud, F., Fauve, S., Odier, P. and Pinton, J.-F., Open questions about homogeneous fluid dynamos; the VKS experiment. Magnetohydrodynamics, 2002, 38, 163-176.

Nore, C., Brachet, M., Politano, H. and Pouquet, A., Dynamo action in the Taylor-Green vortex near threshold. Phys. Plasmas, 1997, 4, 1-4.

Plunian, F., Marty, P. and Alemany, A., Kinematic dynamo action in a network of screw motions; application to the core of a fast breeder reactor. J. Fluid Mech., 1999, 382, 137-154.

Ponomarenko, Yu.B., On the theory of hydromagnetic dynamos. Zh. Prikl. Mekh. \& Tekh. Fiz., 1973, 6, 47-51.

Ponty, Y., Gilbert, A.D. and Soward, A.M., Kinematic dynamo action in large magnetic Reynolds number flows driven by shear and convection. J. Fluid Mech., 2001, 435, 261-287.

Ponty, Y., Gilbert, A.D. and Soward, A.M., The onset of thermal convection in Ekman-Couette shear flow with oblique rotation. J. Fluid Mech., 2003, 487, 91-123.

Ponty, Y., Minnini, P., Pouquet, A., Politano, H., Montgomery, D. and Pinton, J.-F., Numerical study of dynamo action at low magnetic Prandtl numbers. Phys. Rev. Lett., 2005, 94, article 164512.

Roberts, G.O., Dynamo action of fluid motions with two-dimensional periodicity. Phil. Trans. R. Soc. Lond. A, 1972, 271, 411-454.

Ruzmaikin, A.A., Sokoloff, D.D. and Shukurov, A.M., A hydromagnetic screw dynamo. J. Fluid Mech., 1988, 197, 39-56.

Solovyev, A.A., Excitation of magnetic field by the axisymmetric motion of a conducting fluid. Izv. Akad. Nauk USSR, Fiz. Zemli, 1988, 101-103.

Soward, A.M., Fast dynamo action in steady flow. J. Fluid Mech., 1987, 180, 267-295.

Soward, A.M., A unified approach to a class of slow dynamos. Geophys. Astrophys. Fluid Dynam., 1990, 53, 81-107.

Soward, A.M. and Childress, S., Large magnetic Reynolds number dynamo action in spatially periodic flow with mean motion. Phil. Trans. R. Soc. Lond. A, 1990, 331, 649-733.

Stieglitz, R. and Müller, U., Experimental demonstration of a homogeneous two-scale dynamo. Phys. Fluids, 2001, 13, 561-564.

Zhang, P. and Gilbert, A.D., Nonlinear dynamo action in hydrodynamic instabilities driven by shear. Geophys. Astrophys. Fluid Dynam., 2005 (Submitted). 